RESEARCH ARTICLE

Pranay Goswami · Serap Bulut • Teodor Bulboacă

\title{
Certain properties of a new subclass of close-to-convex functions
}

Received: 18 November 2011 / Accepted: 12 March 2012 / Published online: 11 April 2012

(C) The Author(s) 2012. This article is published with open access at Springerlink.com

Abstract In the present paper we introduce and investigate an interesting subclass $\mathcal{K}_{s}^{(k)}(\lambda, h)$ of analytic and close-to-convex functions in the open unit disk $\mathbb{U}$. For functions belonging to the class $\mathcal{K}_{s}^{(k)}(\lambda, h)$, we derive several properties as the inclusion relationships and distortion theorems. The various results presented here would generalize many known recent results.

Mathematics Subject Classification (2010) 30C45 · 30C 80

الملذص

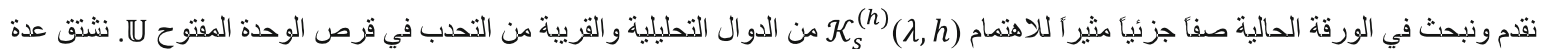

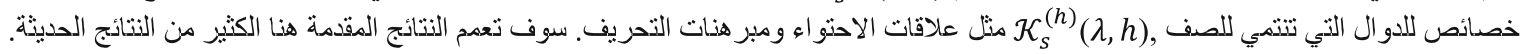

\section{Introduction and preliminaries}

Let $\mathcal{S}$ denote the class of functions of the form

$$
f(z)=z+\sum_{n=2}^{\infty} a_{n} z^{n},
$$

which are univalent (i.e. analytic and injective) in the open unit disk $\mathbb{U}=\{z \in \mathbb{C}:|z|<1\}$. Let $\mathcal{K}$ and $\mathcal{S}^{*}$ denote the usual subclasses of $\mathcal{S}$ whose members are close-to-convex and starlike in $\mathbb{U}$, respectively. We also denote by $\mathcal{S}^{*}(\alpha)$ the class of starlike functions of order $\alpha, 0 \leq \alpha<1$.

\footnotetext{
P. Goswami $(\varangle)$

Department of Mathematics, Amity University Rajasthan,

Jaipur 302003, India

E-mail: pranaygoswami83@gmail.com

S. Bulut

Civil Aviation College, Kocaeli University,

Arslanbey Campus, 41285 Kocaeli, Turkey

E-mail: serap.bulut@kocaeli.edu.tr

T. Bulboacă

Faculty of Mathematics and Computer Science, Babeş-Bolyai University,

400084 Cluj-Napoca, Romania

E-mail: bulboaca@math.ubbcluj.ro
} 
In many earlier investigations, various interesting subclasses of the class $\mathcal{S}$ have been studied from a number of different view points. In particular, Gao and Zhou [2] introduced the next subclass $\mathcal{K}_{s}$ of analytic functions, which is indeed a subclass of close-to-convex functions:

Definition 1.1 [2] Let the function $f$ be analytic in $\mathbb{U}$ and normalized by the condition (1.1). We say that $f \in \mathcal{K}_{s}$, if there exists a function $g \in \mathcal{S}^{*}\left(\frac{1}{2}\right)$ such that

$$
\operatorname{Re} \frac{-z^{2} f^{\prime}(z)}{g(z) g(-z)}>0, \quad z \in \mathbb{U} .
$$

In a very recent paper of Şeker [6], it is introduced the following class $\mathcal{K}_{s}^{k}(\gamma)$ :

Definition 1.2 [6] Let the function $f$ be analytic in $\mathbb{U}$ and normalized by the condition (1.1). We say that $f \in \mathcal{K}_{s}^{(k)}(\gamma)(0 \leq \gamma<1)$, if there exists a function $g \in \mathcal{S}^{*}\left(\frac{k-1}{k}\right)(k \in \mathbb{N}$ is a fixed integer $)$ such that

$$
\operatorname{Re} \frac{z^{k} f^{\prime}(z)}{g_{k}(z)}>\gamma, \quad z \in \mathbb{U},
$$

where $g_{k}$ is defined by the equality

$$
g_{k}(z)=\prod_{\nu=0}^{k-1} \varepsilon^{-v} g\left(\varepsilon^{\nu} z\right), \quad \text { where } \varepsilon=e^{2 \pi i / k} .
$$

For $k=2$ we get the class $\mathcal{K}_{s}(\gamma) \equiv \mathcal{K}_{s}^{(2)}(\gamma)$, introduced and studied by Kowalczyk and Leś-Bomba [3]. Also, for $k=2$ and $\gamma=0$ we obtain the class $\mathcal{K}_{s}^{(2)}(0) \equiv \mathcal{K}_{s}(0) \equiv \mathcal{K}_{s}$ given in the Definition 1.1.

Definition 1.3 (see, e.g. [5]) For two functions $f$ and $g$ analytic in $\mathbb{U}$, we say that the function $f$ is subordinate to $g$, and write $f(z) \prec g(z)$, if there exists a Schwarz function $w$, which (by definition) is analytic in $\mathbb{U}$, with $w(0)=0$, and $|w(z)|<1$ for all $z \in \mathbb{U}$, such that

$$
f(z)=g(w(z)), \quad z \in \mathbb{U} .
$$

In particular, if the function $g$ is univalent in $\mathbb{U}$, then above subordination is equivalent to

$$
f(0)=g(0) \text { and } f(\mathbb{U}) \subset g(\mathbb{U}) .
$$

Motivated by the aforementioned works we now introduce the following subclass of analytic functions:

Definition 1.4 Let $h: \mathbb{U} \rightarrow \mathbb{C}$ be a convex function such that

$$
h(0)=1, \quad h(\bar{z})=\overline{h(z)}, \quad \text { and } \quad \operatorname{Re} h(z)>0, z \in \mathbb{U} .
$$

Suppose also that the function $h$ satisfies the following conditions for all $r \in(0,1)$ :

$$
\begin{aligned}
& \min \{|h(z)|:|z|=r\}=\min \{h(r), h(-r)\}, \\
& \max \{|h(z)|:|z|=r\}=\max \{h(r), h(-r)\} .
\end{aligned}
$$

Let the function $f$ be analytic in $\mathbb{U}$ and normalized by the condition (1.1). We say that $f \in \mathcal{K}_{s}^{(k)}(\lambda, h)$, if there exists a function $g \in \mathcal{S}^{*}\left(\frac{k-1}{k}\right)(k \in \mathbb{N}$ is a fixed integer), such that

$$
\frac{z^{k} f^{\prime}(z)+\lambda z^{k+1} f^{\prime \prime}(z)}{g_{k}(z)} \in h(\mathbb{U}), z \in \mathbb{U}, \quad \text { where } 0 \leq \lambda \leq 1 .
$$

For $\lambda=0$ and $k=2$ we obtain the class $\mathcal{K}_{s}(h) \equiv \mathcal{K}_{s}^{(2)}(0, h)$, recently studied by Xu et al. [11].

Remark 1.1 There are many choices of the function $h$ which would provide interesting subclasses of analytic functions. 
(i) If we let

$$
h(z)=\frac{1+A z}{1+B z} \quad(-1 \leq B<A \leq 1),
$$

then it is easy to verify that $h$ is a convex function in $\mathbb{U}$, and satisfies the hypothesis of Definition 1.4. If $f \in \mathcal{K}_{s}^{(k)}(\lambda, h)$, then

$$
\frac{z^{k} f^{\prime}(z)+\lambda z^{k+1} f^{\prime \prime}(z)}{g_{k}(z)} \prec \frac{1+A z}{1+B z},
$$

where $0 \leq \lambda \leq 1,-1 \leq B<A \leq 1$, and $g \in \mathcal{S}^{*}\left(\frac{k-1}{k}\right)(k \in \mathbb{N}$ is a fixed integer), and this class will be denoted by $\mathcal{K}_{s}^{(k)}(\lambda, A, B)$.

For $k=2$ we have the class $\mathcal{K}_{s}(\lambda, A, B) \equiv \mathcal{K}_{s}^{(2)}(\lambda, A, B)$, recently studied by Wang and Chen [7].

(ii) For

$$
h(z)=\frac{1+(1-2 \gamma) z}{1-z} \quad(0 \leq \gamma<1)
$$

we get the new class $\mathcal{K}_{s}^{(k)}(\lambda, \gamma) \equiv \mathcal{K}_{s}^{(k)}(\lambda, A, B)$, which consists of the functions $f$ that are analytic in $\mathbb{U}$ and normalized by the condition (1.1), satisfying

$$
\operatorname{Re} \frac{z^{k} f^{\prime}(z)+\lambda z^{k+1} f^{\prime \prime}(z)}{g_{k}(z)}>\gamma, \quad z \in \mathbb{U}
$$

Also, for $\gamma=0$ we obtain the new class $\mathcal{K}_{s}^{(k)}(\lambda) \equiv \mathcal{K}_{s}^{(k)}(\lambda, \gamma)$ which consists of the functions $f$ that are analytic in $\mathbb{U}$ and normalized by the condition (1.1), satisfying

$$
\operatorname{Re} \frac{z^{k} f^{\prime}(z)+\lambda z^{k+1} f^{\prime \prime}(z)}{g_{k}(z)}>0, \quad z \in \mathbb{U} .
$$

(iii) Letting $\lambda=0$ in (1.7) we get the class $\mathcal{K}_{s}^{(k)}(\gamma)$ given in Definition 1.2.

In this work, by using the principle of subordination, we obtain inclusion theorem and distortion theorems for functions in the function class $\mathcal{K}_{s}^{(k)}(\lambda, h)$. Our results unify and extend the corresponding results obtained by Xu et al. [11], Wang and Chen [7], Wang et al. [8,9], Şeker [6], Kowalczyk and Leś-Bomba [3], and Gao and Zhou [2].

\section{Main results}

We assume throughout this section that $k \in \mathbb{N}$ is a fixed integer.

In order to prove our main results for the functions class $\mathcal{K}_{s}^{(k)}(\lambda, h)$, we first recall the following lemmas.

Lemma 2.1 ([8, Theorem 2]) If $g(z)=z+\sum_{n=2}^{\infty} b_{n} z^{n} \in \mathcal{S}^{*}\left(\frac{k-1}{k}\right)$, then

$$
G_{k}(z)=\frac{g_{k}(z)}{z^{k-1}}=z+\sum_{n=2}^{\infty} B_{n} z^{n} \in \mathcal{S}^{*} \subset \mathcal{S} .
$$

Lemma 2.2 ([4]) If $\operatorname{Re} \gamma \geq 0$, then $f \in \mathcal{K}$ implies

$$
H(z)=\frac{1+\gamma}{z^{\gamma}} \int_{0}^{z} t^{\gamma-1} f(t) \mathrm{d} t \in \mathcal{K} .
$$

We also mention that the above lemma is a special case of Theorem 4 obtained by $\mathrm{Wu}$ [10].

We now state and prove the main results of our present investigation: 
Theorem 2.1 Let $f$ be an analytic function in $\mathbb{U}$ and normalized by the condition (1.1). Then, $f \in \mathcal{K}_{s}^{(k)}(\lambda, h)$ if and only if there exists a function $g \in \mathcal{S}^{*}\left(\frac{k-1}{k}\right)$, such that

$$
\frac{z^{k} f^{\prime}(z)+\lambda z^{k+1} f^{\prime \prime}(z)}{g_{k}(z)} \prec h(z) \quad(0 \leq \lambda \leq 1)
$$

where $g_{k}$ is given by (1.2).

Proof This result can be proven fairly easily by using the Definition 1.3 combined with the definition inequality (1.7).

In view of the Remark 1.1, if we set $\lambda=0$ and

$$
h(z)=\frac{1+(1-2 \gamma) z}{1-z} \quad(0 \leq \gamma<1)
$$

in Theorem 2.1, we deduce the following corollary:

Corollary 2.1 Let $f$ be an analytic function in $\mathbb{U}$ and normalized by the condition (1.1). Then, $f \in \mathcal{K}_{s}^{(k)}(\gamma)(0 \leq$ $\gamma<1)$ if and only if there exists a function $g \in \mathcal{S}^{*}\left(\frac{k-1}{k}\right)$ such that

$$
\frac{z^{k} f^{\prime}(z)}{g_{k}(z)} \prec \frac{1+(1-2 \gamma) z}{1-z},
$$

where $g_{k}$ is given by (1.2).

Note that Corollary 2.1 was proven by Şeker [6, Theorem 1]. However, by using Theorem 2.1 we are able to deduce this result as an easy consequence of the theorem.

Theorem 2.2 If $0 \leq \lambda \leq 1$, then

$$
\mathcal{K}_{s}^{(k)}(\lambda, h) \subset \mathcal{K} \subset \mathcal{S}^{*}
$$

Proof Let $f \in \mathcal{K}_{s}^{(k)}(\lambda, h)$ be an arbitrary function, and let define the corresponding functions $F$ and $G_{k}$ by

$$
F(z):=(1-\lambda) f(z)+\lambda z f^{\prime}(z), \quad \text { and } \quad G_{k}(z):=\frac{g_{k}(z)}{z^{k-1}} .
$$

Then, the condition (2.2) can be written as

$$
\frac{z F^{\prime}(z)}{G_{k}(z)} \prec h(z)
$$

By Lemma 2.1 we have $G_{k} \in \mathcal{S}^{*}$, and from the above subordination combined with the fact that $\operatorname{Re} h(z)>0$ for all $z \in \mathbb{U}$, we deduce that

$$
F(z)=(1-\lambda) f(z)+\lambda z f^{\prime}(z) \in \mathcal{K} .
$$

Now we will consider the following two cases:

Case 1. If $\lambda=0$, then it is obvious that $f=F \in \mathcal{K}$.

Case 2. If $0<\lambda \leq 1$, according to the definition of $F$ we have

$$
f(z)=\frac{1}{\lambda} z^{1-\frac{1}{\lambda}} \int_{0}^{z} t^{\frac{1}{\lambda}-2} F(t) \mathrm{d} t .
$$

Denoting $\gamma=1 / \lambda-1$, then $\operatorname{Re} \gamma \geq 0$, and by using Lemma 2.2 we conclude that $f \in \mathcal{K}$, which complete the proof of our theorem. 
Theorem 2.3 Suppose that the convex function $h: \bar{U} \rightarrow \mathbb{C}$ satisfy the conditions (1.3) and (1.4), and $g \in \mathcal{S}^{*}\left(\frac{k-1}{k}\right)$, where $g_{k}$ is given by (1.2).

Let $f$ be an analytic function in $\mathbb{U}$ of the form (1.1). Then, $f \in \mathcal{K}_{s}^{(k)}(\lambda, h)$ if and only if

$$
1+\sum_{n=2}^{\infty} \frac{n+\lambda n(n-1)}{1-h\left(e^{i \theta}\right)} a_{n} z^{n-1}-\sum_{n=2}^{\infty} \frac{h\left(e^{i \theta}\right)}{1-h\left(e^{i \theta}\right)} B_{n} z^{n-1} \neq 0,
$$

for all $z \in \mathbb{U}$ and $\theta \in[0,2 \pi)$, where the coefficients $B_{n}$ are given by (2.1).

Proof Since $g \in \mathcal{S}^{*}\left(\frac{k-1}{k}\right)$, then $g$ is univalent in $\mathbb{U}$, hence it follows that $g_{k}(z)=\prod_{\nu=0}^{k-1} \varepsilon^{-v} g\left(\varepsilon^{v} z\right) \neq 0$ for all $z \in \mathbb{U} \backslash\{0\}$, that is

$$
\frac{g_{k}(z)}{z^{k}} \neq 0, \quad z \in \mathbb{U}
$$

(i) First, supposing that $f \in \mathcal{K}_{s}^{(k)}(\lambda, h)$, from (2.2) we have

$$
\frac{z^{k} f^{\prime}(z)+\lambda z^{k+1} f^{\prime \prime}(z)}{g_{k}(z)} \prec h(z) .
$$

From here, according to the definition of the subordination of two functions, there exists a function $w$, which is analytic in $\mathbb{U}$, with $w(0)=0$, and $|w(z)|<1, z \in \mathbb{U}$, such that

$$
\frac{z^{k} f^{\prime}(z)+\lambda z^{k+1} f^{\prime \prime}(z)}{g_{k}(z)} \prec h(w(z))
$$

and thus

$$
\frac{z^{k} f^{\prime}(z)+\lambda z^{k+1} f^{\prime \prime}(z)}{g_{k}(z)} \neq h\left(e^{i \theta}\right), z \in \mathbb{U}, \theta \in[0,2 \pi) .
$$

According to (2.4) and using the fact that $h$ is univalent in $\mathbb{U}$, the previous subordination is equivalent to

$$
f^{\prime}(z)+\lambda z f^{\prime \prime}(z) \neq h\left(e^{i \theta}\right) \frac{g_{k}(z)}{z^{k}}, \quad z \in \mathbb{U}, \quad \theta \in[0,2 \pi) .
$$

and according to Lemma 2.1 the above relation leads to (2.3), hence it proves the first part of our result.

(ii) Reversely, since it was previously shown that the assumption (2.3) is equivalent to (2.6), using (2.4) we obtain that

$$
\frac{z^{k} f^{\prime}(z)+\lambda z^{k+1} f^{\prime \prime}(z)}{g_{k}(z)} \neq h\left(e^{i \theta}\right), z \in \mathbb{U}, \theta \in[0,2 \pi) .
$$

If we denote

$$
\varphi(z)=\frac{z^{k} f^{\prime}(z)+\lambda z^{k+1} f^{\prime \prime}(z)}{g_{k}(z)},
$$

the relation (2.7) shows that $\varphi(\mathbb{U}) \cap h(\partial \mathbb{U})=\emptyset$. Thus, the simply-connected domain $\varphi(\mathbb{U})$ is included in a connected component of $\mathbb{C} \backslash h(\partial \mathbb{U})$. From here, using the fact that $\varphi(0)=h(0)$ together with the univalence of the function $h$, it follows that $\varphi(z) \prec h(z)$, which represents in fact the subordination (2.5), i.e. $f \in \mathcal{K}_{s}^{(k)}(\lambda, h)$.

For the special case when the function $h$ is given by (1.6), from Theorem 2.3 we obtain the following result:

Corollary 2.2 Suppose that $g \in \mathcal{S}^{*}\left(\frac{k-1}{k}\right)$, and $g_{k}$ is given by (1.2). If $f$ is an analytic function in $\mathbb{U}$ of the form (1.1), such that

$$
(1+|B|) \sum_{n=2}^{\infty}[n+\lambda n(n-1)]\left|a_{n}\right|+(1+|A|) \sum_{n=2}^{\infty}\left|B_{n}\right|<A-B,
$$

where the coefficients $B_{n}$ are given by (2.1), then $f \in \mathcal{K}_{s}^{(k)}(\lambda, A, B)$. 
Proof For the function $h$ given by (1.6), the above theorem yields that $f \in \mathcal{K}_{s}^{(k)}(\lambda, A, B)$ if and only if

$$
1+\sum_{n=2}^{\infty} \frac{[n+\lambda n(n-1)]\left(B+e^{-i \theta}\right)}{B-A} a_{n} z^{n-1}-\sum_{n=2}^{\infty} \frac{A+e^{-i \theta}}{B-A} B_{n} z^{n-1} \neq 0
$$

for all $z \in \mathbb{U}$ and $\theta \in[0,2 \pi)$. Now, a simple computation combined with the assumption (2.8) shows that

$$
\begin{aligned}
\mid & +\sum_{n=2}^{\infty} \frac{[n+\lambda n(n-1)]\left(B+e^{-i \theta}\right)}{B-A} a_{n} z^{n-1}-\sum_{n=2}^{\infty} \frac{A+e^{-i \theta}}{B-A} B_{n} z^{n-1} \mid \\
& \geq 1-\left|\sum_{n=2}^{\infty} \frac{[n+\lambda n(n-1)]\left(B+e^{-i \theta}\right)}{B-A} a_{n} z^{n-1}\right|-\left|\sum_{n=2}^{\infty} \frac{A+e^{-i \theta}}{B-A} B_{n} z^{n-1}\right| \\
& \geq 1-\sum_{n=2}^{\infty} \frac{[n+\lambda n(n-1)](|B|+1)}{A-B}\left|a_{n}\right|-\sum_{n=2}^{\infty} \frac{|A|+1}{A-B}\left|B_{n}\right|>0,
\end{aligned}
$$

for all $z \in \mathbb{U}$ and $\theta \in[0,2 \pi)$, hence (2.9) holds.

Substituting $A=1-2 \gamma(0 \leq \gamma<1)$ and $B=-1$ in the above corollary, we obtain the following special case:

Corollary 2.3 Suppose that $g \in \mathcal{S}^{*}\left(\frac{k-1}{k}\right)$, and $g_{k}$ is given by (1.2). If $f$ is an analytic function in $\mathbb{U}$ of the form (1.1), such that

$$
2 \sum_{n=2}^{\infty}[n+\lambda n(n-1)]\left|a_{n}\right|+(1+|1-2 \gamma|) \sum_{n=2}^{\infty}\left|B_{n}\right|<2(1-\gamma) .
$$

where the coefficients $B_{n}$ are given by (2.1), then $f \in \mathcal{K}_{s}^{(k)}(\lambda, \gamma)$.

Remark 2.1 (i) Putting $A=\beta, B=-\alpha \beta(0 \leq \alpha \leq 1,0<\beta \leq 1)$, and $\lambda=0$ in Corollary 2.2, we get a known result obtained by Wang et al. [8].

(ii) Letting $\lambda=0$ in Corollary 2.3 we obtain the result given by Şeker [6].

Theorem 2.4 If $f \in \mathcal{K}_{s}^{(k)}(\lambda, h)$, then:

(i) if $0 \leq \lambda \leq 1$, for $|z| \leq r(0 \leq r<1)$, we have

$$
\frac{\min \{h(r), h(-r)\}}{(1+r)^{2}} \leq\left|f^{\prime}(z)+\lambda z f^{\prime \prime}(z)\right| \leq \frac{\max \{h(r), h(-r)\}}{(1-r)^{2}}
$$

(ii) for $|z| \leq r(0 \leq r<1)$, we have

$$
\int_{0}^{r} \frac{\min \{h(t), h(-t)\}}{(1+t)^{2}} \mathrm{~d} t \leq|f(z)| \leq \int_{0}^{r} \frac{\max \{h(t), h(-t)\}}{(1-t)^{2}} \mathrm{~d} t ;
$$

(iii) if $0<\lambda \leq 1$, for $|z| \leq r(0 \leq r<1)$, we have

$$
\begin{aligned}
& \frac{1}{\lambda} r^{1-\frac{1}{\lambda}} \int_{0}^{r} \int_{0}^{s} \frac{\min \{h(t), h(-t)\}}{(1+t)^{2}} s^{\frac{1}{\lambda}-2} \mathrm{~d} s \mathrm{~d} t \leq|f(z)| \\
& \leq \frac{1}{\lambda} r^{1-\frac{1}{\lambda}} \int_{0}^{r} \int_{0}^{s} \frac{\max \{h(t), h(-t)\}}{(1-t)^{2}} s^{\frac{1}{\lambda}-2} \mathrm{~d} s \mathrm{~d} t .
\end{aligned}
$$


Proof Since $f \in \mathcal{K}_{s}^{(k)}(\lambda, h)$, there exists a function $g \in \mathcal{S}^{*}\left(\frac{k-1}{k}\right)$ such that (1.5) holds. From Lemma 2.1 it follows that the function $G_{k}$ given by (2.1) is starlike, and according to the well-known inequalities [1, p. 70] we have

$$
\frac{r}{(1+r)^{2}} \leq\left|G_{k}(z)\right| \leq \frac{r}{(1-r)^{2}}, \quad|z| \leq r(0 \leq r<1) .
$$

From the definition (1.5) combined with (1.4), we deduce that

$$
\min \{h(r), h(-r)\} \leq\left|\frac{z f^{\prime}(z)+\lambda z^{2} f^{\prime \prime}(z)}{G_{k}(z)}\right| \leq \max \{h(r), h(-r)\}, \quad|z| \leq r .
$$

Letting

$$
F(z)=(1-\lambda) f(z)+\lambda z f^{\prime}(z),
$$

then $F^{\prime}(z)=f^{\prime}(z)+\lambda z f^{\prime \prime}(z)$, and the inequality (2.14) may be written as

$$
\min \{h(r), h(-r)\} \leq\left|\frac{z F^{\prime}(z)}{G_{k}(z)}\right| \leq \max \{h(r), h(-r)\}, \quad|z| \leq r .
$$

From (2.13) and (2.16) we obtain that

$$
\frac{\min \{h(r), h(-r)\}}{(1+r)^{2}} \leq\left|F^{\prime}(z)\right| \leq \frac{\max \{h(r), h(-r)\}}{(1-r)^{2}}, \quad|z| \leq r,
$$

which proves (2.10).

If $\ell$ denotes the closed line-segment that connects the points 0 and $z=r e^{i \theta}(0 \leq r<1)$, i.e. $\ell=\left[0, r e^{i \theta}\right]$, then

$$
|F(z)|=\left|\int_{\ell} F^{\prime}(\zeta) \mathrm{d} \zeta\right|=\left|\int_{0}^{r} F^{\prime}\left(t e^{i \theta}\right) e^{i \theta} \mathrm{d} t\right| \leq \int_{0}^{r}\left|F^{\prime}\left(t e^{i \theta}\right)\right| \mathrm{d} t,
$$

and from the right-hand side part of (2.17) we deduce that

$$
|F(z)| \leq \int_{0}^{r} \frac{\max \{h(t), h(-t)\}}{(1-t)^{2}} \mathrm{~d} t, \quad|z|=r .
$$

Since $f \in \mathcal{K}_{s}^{(k)}(\lambda, h)$, then

$$
\frac{z F^{\prime}(z)}{G_{k}(z)} \prec h(z)
$$

where $G_{k} \in \mathcal{S}^{*}$, and $\operatorname{Re} h(z)>0$ for all $z \in \mathbb{U}$. Thus, we deduce that $F \in \mathcal{K}$, hence the function $F$ is univalent in $\mathbb{U}$.

To prove the corresponding left-hand side inequality, let $z_{0} \in \mathbb{U}$ with $\left|z_{0}\right|=r$, such that

$$
\left|F\left(z_{0}\right)\right|=\min \{|F(z)|:|z|=r\},
$$

for some $0<r<1$. It is sufficient to prove that the left-hand side inequality holds for this point $z_{0}$, because, otherwise, we have $|F(z)| \geq\left|F\left(z_{0}\right)\right|$ for all $|z|=r$. Since the function $F$ is univalent in $\mathbb{U}$, the image of the closed line-segment $\sigma=\left[0, F\left(z_{0}\right)\right]$ by $F^{-1}$ is a simple Jordan curve $\Lambda$ included in the closed disk $\{z \in \mathbb{C}:|z| \leq r\}$, i.e. $\Lambda=F^{-1}(\sigma) \subset\{z \in \mathbb{C}:|z| \leq r\}$. 
Let denote $z_{0}=r e^{i \theta}$, and $F\left(z_{0}\right)=R e^{i \Phi}$. If $w \in \sigma$ is an arbitrary point, then $w=s e^{i \Phi}$, where $s \in[0, R]$, hence $|\mathrm{d} w|=\mathrm{d} s$. Denoting $\zeta=F^{-1}(w)$, then $\zeta=t e^{i \varphi}$, hence $\mathrm{d} \zeta=e^{i \varphi} \mathrm{d} t+t i e^{i \varphi} \mathrm{d} \varphi$, and thus $|\mathrm{d} \zeta| \geq \mathrm{d} t$. From here and from the left-hand side inequality of (2.17), it follows that

$$
\begin{aligned}
\left|F\left(z_{0}\right)\right| & =\left|F\left(r e^{i \theta}\right)\right|=\int_{0}^{R} \mathrm{~d} s=\int_{\sigma}|\mathrm{d} w|=\int_{\Lambda}\left|F^{\prime}(\zeta)\right||\mathrm{d} \zeta| \\
& \geq \int_{0}^{r}\left|F^{\prime}\left(t e^{i \varphi}\right)\right| \mathrm{d} t \geq \int_{0}^{r} \frac{\min \{h(t), h(-t)\}}{(1+t)^{2}} \mathrm{~d} t,
\end{aligned}
$$

hence

$$
|F(z)| \geq \int_{0}^{r} \frac{\min \{h(t), h(-t)\}}{(1+t)^{2}} \mathrm{~d} t, \quad|z|=r .
$$

Combining the inequalities (2.18) and (2.19), together with the maximum modulus principle, we have

$$
\begin{aligned}
\int_{0}^{r} \frac{\min \{h(t), h(-t)\}}{(1+t)^{2}} \mathrm{~d} t & \leq\left|(1-\lambda) f(z)+\lambda z f^{\prime}(z)\right| \\
& \leq \int_{0}^{r} \frac{\max \{h(t), h(-t)\}}{(1-t)^{2}} \mathrm{~d} t, \quad|z| \leq r .
\end{aligned}
$$

To complete our proof, we will discuss the following two cases for the parameter $\lambda \in[0,1]$ :

Case 1. For $\lambda=0$, from (2.20) we easily get (2.11).

Case 2. For $0<\lambda \leq 1$, from (2.15) we obtain

$$
f(z)=\frac{1}{\lambda} z^{1-\frac{1}{\lambda}} \int_{0}^{z} t^{\frac{1}{\lambda}-2} F(t) \mathrm{d} t,
$$

hence we easily conclude that (2.12) holds.

Remark 2.2 The results obtained by Xu et al. [11, Theorem 3], Wang and Chen [7, Theorem 5.1], Şeker [6, Theorem 4], and Kowalczyk and Leś-Bomba [3, Theorem 4] are special cases of our Theorem 2.4.

Acknowledgments The first author (Pranay Goswami) is grateful to Prof. S.P. Goyal, Emeritus Scientist, Department of Mathematics, University of Rajasthan, Jaipur, India, for his guidance and constant encouragement.

Open Access This article is distributed under the terms of the Creative Commons Attribution License which permits any use, distribution, and reproduction in any medium, provided the original author(s) and the source are credited.

\section{References}

1. Duren, P.L.: Univalent Functions. Grundlehren der Mathematischen Wissenschaften 259. Springer, New York, Berlin, Heidelberg, Tokyo (1983)

2. Gao, C.-Y.; Zhou, S.-Q.: On a class of analytic functions related to the starlike functions. Kyungpook Math. J. 45, 123-130 (2005)

3. Kowalczyk, J.; Leś-Bomba, E.: On a subclass of close-to-convex functions. Appl. Math. Lett. 23, 1147-1151 (2010)

4. Lewandowski, Z.; Miller, S.S.; Złotkiewicz, E.: Generating functions for some classes of univalent functions. Proc. Am. Math. Soc. 56, 111-117 (1976)

5. Miller, S.S.; Mocanu, P.T.: Differential Subordinations. Theory and Applications. Series on Monographs and Textbooks in Pure and Applied Mathematics, vol. 225. Marcel Dekker, New York (2000)

6. Şeker, B.: On certain new subclass of close-to-convex functions. Appl. Math. Comput. 218(3), 1041-1045 (2011)

7. Wang, Z.-G.; Chen, D.-Z.: On a subclass of close-to-convex functions. Hacet. J. Math. Stat. 38(2), 95-101 (2009) 
8. Wang, Z.-G.; Gao, C.-Y.; Yuan, S.-M.: On certain new subclass of close-to-convex functions. Mat. Vesnik 58, 119-124 (2006)

9. Wang, Z.-G.; Gao, C.-Y.; Yuan, S.-M.: On certain subclass of close-to-convex functions. Acta Math. Acad. Paedagog. Nyházi. (N.S.) 22, 171-177 (2006)

10. $\mathrm{Wu}, \mathrm{Z}$.-R.: The integral operator of starlikeness and the family of Bazilević functions. Acta Math. Sin. 27, 394-409 (1984, in Chinese)

11. Xu, Q.-H.; Srivastva, H.M.; Li, Z.: On certain subclass of analytic and close-to-convex functions. Appl. Math. Lett. 24, 396-401 (2011) 